(C) The Author(s)

DOI: $10.1142 / \mathrm{S} 2010194516602088$

\title{
Strategy for the engineering integration of the ESS accelerator
}

\author{
Nikolaos Gazis \\ Accelerator Division, European Spallation Source, P.O Box 176 \\ Lund, 22100, Sweden \\ nick.gazis@esss.se \\ David McGinnis \\ Accelerator Division, European Spallation Source, P.O Box 176 \\ Lund, 22100, Sweden \\ david.mcginnis@esss.se \\ Stephen Molloy \\ Accelerator Division, European Spallation Source, P.O Box 176 \\ Lund, 22100, Sweden \\ stephen.molloy@esss.se \\ Eugene Tanke \\ Accelerator Division, European Spallation Source, P.O Box 176 \\ Lund, 22100, Sweden \\ eugene.tanke@esss.se \\ Carl-Johan Hardh \\ Engineering and Integration Support Division, European Spallation Source, P.O Box 176 \\ Lund, 22100, Sweden \\ carljohan.hardh@esss.se \\ Daniel Lundgren \\ Engineering and Integration Support Division, European Spallation Source, P.O Box 176 \\ Lund, 22100, Sweden \\ Daniel.Lundgren@esss.se
}

Published 1 September 2016

The European Spallation Source (ESS), currently under construction in Lund, Sweden, will be the world's most powerful source of neutrons. The goal is to deliver neutrons to users in 2019 and reach full power sometime in the middle of the following decade. One of the key issues for ESS is to develop a strategy, along with the proper innovative tools, to efficiently communicate and smoothly collaborate between divisions and groups inside ESS and with its outside collaborators, so-called In-Kind Contributors (IKC). Technical requirements related to the scope to be delivered are among the most important technical information to be exchanged. This information exchange

This is an Open Access article published by World Scientific Publishing Company. It is distributed under the terms of the Creative Commons Attribution 3.0 (CC-BY) License. Further distribution of this work is permitted, provided the original work is properly cited. 
is facilitated by using a commercial requirements management database that is accessible through the web. The physics multidisciplinary needs are linked with the engineering integration through LinacLego, which is a tool that provides all updated lattice data for the accelerator. The lattice information is then gathered and utilized to control the physical positioning of the mechanical engineering components for the accelerator. The precision for this operation is provided by a dedicated mechanical design skeleton in a Computer Aided Design (CAD) environment. Finally, the realization of all these steps is supervised in detail and continuously evaluated. In this way the required ESS machine design can be delivered, both in terms of the engineering and the physics aspects.

Keywords: Accelerator; Integration; In-Kind; LinacLego.

\section{Introduction}

Construction of the European Spallation Source (ESS), which is a neutron spallation source, started in Lund (Sweden) in June 2014. The ESS will house the most powerful proton linac ever built. Protons will be accelerated in a sequence of normal conducting and superconducting accelerating structures before hitting the neutron rich tungsten target. The $5 \mathrm{MW}$ proton linac has a peak power of $125 \mathrm{MW}$ and will deliver $2 \mathrm{GeV}$ beams with a peak beam current of $62.5 \mathrm{~mA}$. The long pulse length of $2.86 \mathrm{~ms}$ will have a repetition rate of $14 \mathrm{~Hz}$, corresponding to a duty factor of $4 \%$. A high flux of pulsed spallation neutrons from the target will undergo energy regulation in the moderators and will finally reach the instruments where the detectors and experimental stands will reside. The approximately $600 \mathrm{~m}$ long linac will require RF power generated by over 150 individual high power RF sources, which will cost over $200 \mathrm{M} €$. In order for the ESS to be realized, several collaborations have been formed among institutions all over Europe to cover the costs, either by contributing to the budget or via in-kind contributions of parts. The development of innovative strategies and tools for data exchange (CAD, technical specifications and other) and management is fundamental not only to foster new collaborations but also to satisfy the needs and interests of both ESS and its partners.

\section{ESS Accelerator}

The ESS accelerator consists of two major sections: the warm (room temperature) linac and the cold (cryogenic at $2 \mathrm{~K}$ temperature) linac. The different subsystems of the ESS accelerator are presented in Fig. 1.

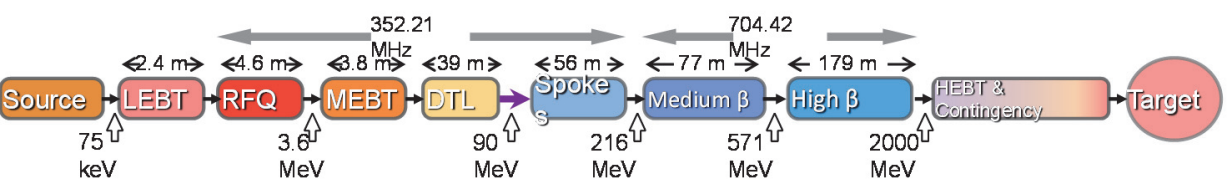

Fig. 1. Schematic layout of the ESS accelerator. ${ }^{1}$

The initial stage of the warm linac, which is $54.3 \mathrm{~m}$ in length, is an Ion Source (ISRC) housed inside a High Voltage (HV) cage of $4.5 \mathrm{~m}$ total length. It extracts protons 
of $75 \mathrm{keV}$ and injects them into a $2.4 \mathrm{~m}$ long Low Energy Beam Transport (LEBT). Solenoid magnets in the LEBT then focus the beam into the $4.6 \mathrm{~m}$ long, Radio Frequency Quadrupole (RFQ) of $352 \mathrm{MHz}$ frequency. In the RFQ, the beam gets bunched and accelerated up to the energy of $3.6 \mathrm{MeV}$ before entering the $3.8 \mathrm{~m}$ long Medium Energy Beam Transport (MEBT). The MEBT is followed by a five-tank $352 \mathrm{MHz}$ Drift Tube Linac (DTL) with output energy of $90 \mathrm{MeV}$.

At this location, the $704 \mathrm{MHz}$ cold linac starts with the so-called Low Energy Differential Pumping (LEDP) station. The beam is then transported to 13 in total Spoke (SPK) Cryomodules (CM) for acceleration. Linac Warm Units (LWU) placed in between each SPK CM, contain quadrupoles to ensure that the beam stays in focus and instrumentation to detect the beam characteristics. Vacuum pumps are also integrated into each LWU. The $56 \mathrm{~m}$ long SPK section provides a beam of $216 \mathrm{MeV}$ to the $77 \mathrm{~m}$ long Medium Beta Linac (MBL). The MBL consists of 9 CMs with LWUs in between to fulfill beam optics requirements and house various instrumentations. The last section of the cold linac is the High Beta Linac (HBL), again with a sequence of $21 \mathrm{CMs}$ followed by LWUs for $179 \mathrm{~m}$, after which the beam reaches the final energy of $2 \mathrm{GeV}$.

The accelerator continues with the High Energy Beam Transport (HEBT) area that has a total straight length of $215 \mathrm{~m}$. The HEBT contains more than $20 \mathrm{LWUs}$, and the Dog-Leg (DgLg) area bends the beam upward with an inclination of $4^{\circ}$ toward the Accelerator to Target (A2T) area. The physical interface between the accelerator and Target Systems is formed by the neutron shield wall. Still, the beam tube will cross the neutron shield wall and will end up on target through a beam delivery window or other similar set-up. Beneath the DgLg, a tuning Beam Dump (BD) is surrounded by roughly 300 tons of steel in order to accommodate a beam power of $12.5 \mathrm{~kW}$.

All accelerator parts for both warm and cold sections will be fed with RF power produced in the Klystron Gallery (KG) that runs parallel to the Accelerator Tunnel (AT). Dedicated RF-waveguides coming from the KG to the AT pass through stubs. These stubs also serve to provide transit for all cabling from the KG to the AT and could house a limited number of shielded instrumentation. The overall ESS physical plant is presented in Fig. 2. The proton beam is generated in the ISRC, which is housed in the Front-End Building (FEB), and reaches the tungsten target through the AT. Several ESS experiments will take place in the Target Building with the majority of the instruments to be installed in the halls of the Instruments Building. 


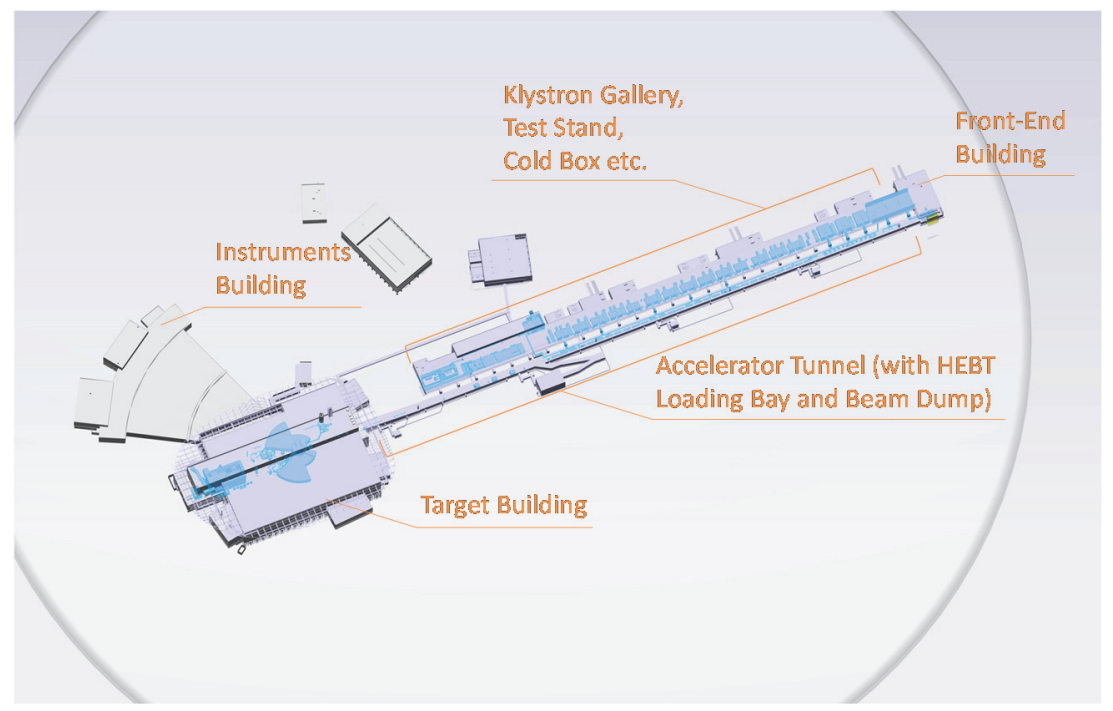

Fig. 2. The buildings for AT, KG, Target, Instruments etc.at the ESS site.

\section{Accelerator Requirements and Definition}

A commercial database for requirements management that is accessible through the web is already in place to facilitate information exchange but also to provide a baseline of the requirements for the components of the ESS accelerator. Requirements are categorized in levels based on a Product Breakdown Structure (PBS). The top-level accelerator requirements derive from the ESS stakeholder requirements. The levels below cover the broad principles that engulf accelerator sections down to the last sub-assembly and accelerator slot and associated devices. The requirements are discussed and agreed upon by the ESS lead engineers, Work Package (WP) leaders, ESS divisions and the IKCs. The WP provides technical specifications derived from the requirements either by servicing principles (e.g. electrical WP) or by defining a vertical accelerator sub-assembly (e.g. warm linac WP).

\section{Accelerator Integration}

The ESS accelerator needs a robust implementation of the dedicated models of the accelerator parts. An integrated model needs to correctly scale the machine and identify any clashes or other potential issues. LinacLego ${ }^{2}$ is the fundamental tool that provides a single-point-of-truth for the tunnel components containing all physics, engineering, CAD and physical plant details. Amongst the technical description and the engineering details for the machine (e.g. optical parameters, energy thresholds, etc.), LinacLego precisely describes the accelerator lattice and documents the lengths for each of the dedicated components, the so-called slot length. The lattice is described in a hierarchy of linac sections constituted by repetitive cells. Each cell is formed by slots. The slots are defined as the modular sub-assemblies that, after installation in sequence, will constitute the 
linac. The majority of the ESS accelerator components inside the slots come from 25 different $\mathrm{IKC}^{3}$ that are located throughout Europe. The last breakdown of the linac is the beamline elements that form the slots. The lattice hierarchy of the accelerator is presented in Fig. 3, with emphasis on the slot level which is used for the information and file exchange between ESS and IKCs.

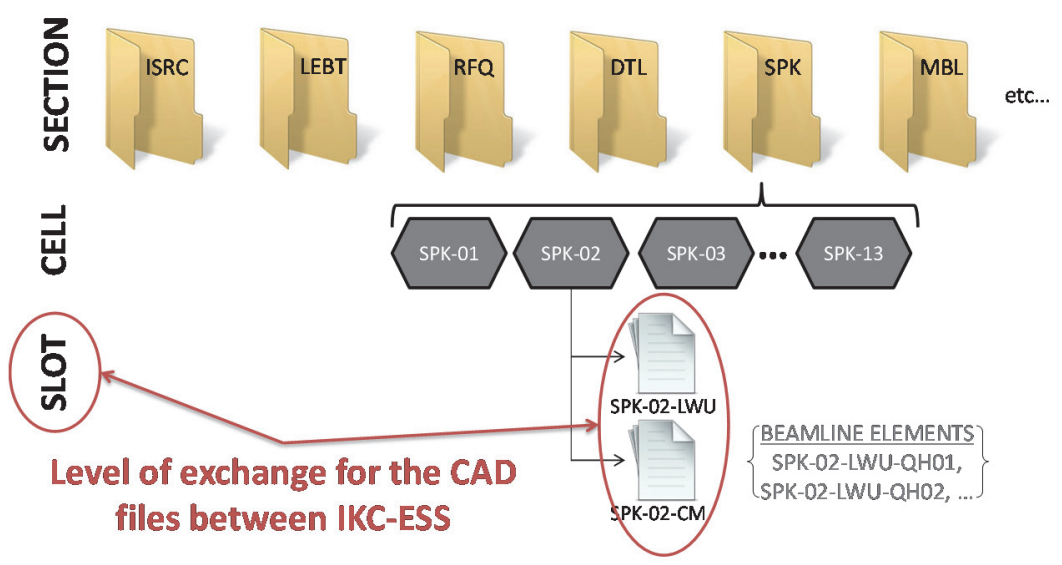

Fig. 3. Lattice hierarchy in LinacLego — Integration Drawing Exchange Solution (IDES).

ESS needs to assimilate all the different parts that are stored and versioned under the Collaboration Home of ESS (CHESS ${ }^{4}$ ) into one master design (CHESS reference ESS0016885). ESS has developed and is utilizing CHESS as the centralized document and design management system. Since the civil construction is already well under way, ESS needs to keep track of the current design status of the slots and provide rapid feedback to the IKCs. Design changes could impact the schedule or inflict changes. Therefore the Accelerator Integration Group (AIG) put in use LinacLego and CHESS and developed the IDES methodology to efficiently exchange CAD and other information with storage safety, versioning and revision control.

By combining the dimensions in LinacLego and the input of IKCs utilizing IDES, the design effort is centralized and led by ESS for the creation of the master skeleton for the $\mathrm{CAD}$ integration. Since the in-kind partners use miscellaneous CAD software, the conversion into CAD of the natively contributed slot files is also handled by the ESS integration team. The back-office conversion operation is well established in the AIG, covering a wide spectrum of input files that derive either from engineering CAD software (mainly used by IKCs) or architectural BIM software, mainly used by ESS Conventional Facilities (CF).

The accelerator skeleton in the CAD master model is defined by the Target Coordinate System (TCS). The Machine Coordinate System (MCS) of the accelerator is defined according to the TCS, and although it is widely utilized (in 2D drawings and site construction), it could potentially be moved relative to the TCS following the machine 
needs. The correlation between the CAD accelerator skeleton and the lattice hierarchy levels of IDES is presented in Fig. 4.

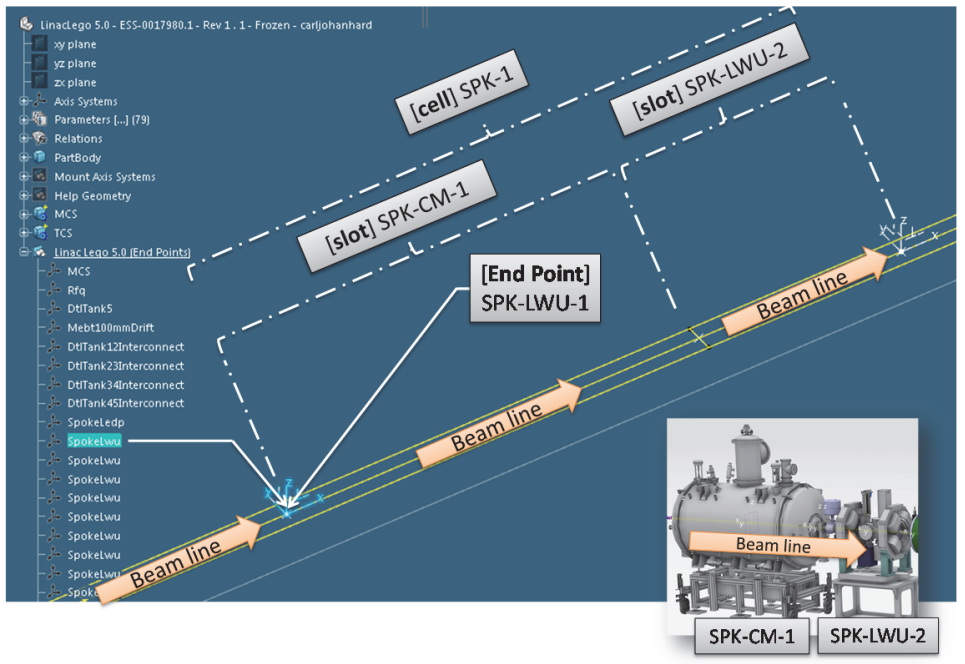

Fig. 4. CAD skeleton according to the lattice hierarchy of LinacLego.

Having set both TCS and MCS and attached to them the linac slots, the rest of the systems, namely BD, cooling skids and racks in the FEB, are positioned and connected to the linac. The routed components (waveguides, cables, pipes, etc.) pass through the stubs to the KG where they hook up to the RF supply, electrical, and electronic equipment that power up and control the linac (klystrons, modulators, power supplies, beam instrumentation, etc.). The KG integration, although not based on a skeleton, indeed is a combination of engineering effort for space optimization, efficient planning and installation sequences, operation (beam-on), and maintenance scenarios. Although the integration of the machine dictates the stub longitudinal positioning (with respect to the beam axis) in the tunnel, on the side of the $\mathrm{KG}$ the stub opening constrains the equipment placement and positioning.

\section{Accelerator Integration Goals}

It is evident that, for the first time, a European linac is being built with such a significant amount of in-kind contribution. Amongst the major goals of the AIG is to set milestones that will allow all parties to respect the selected timeframe, push forward towards the realization of the civil construction, and lay in place the ESS detailed accelerator master model including all the various pieces of information. In parallel, the AIG prepares for the installation and commissioning according to both schedule and master model. The driver of the most powerful neutron source that has ever existed, the integrated linac, is planned to deliver the first protons on target in 2019. 


\section{References}

1. M. Eshraqi et al., The ESS linac, in Proc. IPAC 2014, http://www.jacow.org/,_THPME043, p. 3320.

2. ESS LinacLego, ESS Linac Parameter Book, https://aig.esss.lu.se:8443/LinacLegoWebApp/.

3. D. McGinnis, New design approaches for high intensity superconducting linacs - the new ESS linac design, in Proc. IPAC 2014, http://www.jacow.org/, MOYBA02 THPME043, p. 23.

4. Collaboration Home of ESS, CHESS, https://chess.esss.lu.se/. 